\title{
Erratum to: A Review of Technology-Based Interventions to Teach Academic Skills to Students with Autism Spectrum Disorder
}

Victoria Knight • Bethany R. McKissick • Alicia Saunders

Published online: 17 May 2013

(C) Springer Science+Business Media New York 2013

Erratum to: J Autism Dev Disord

DOI 10.1007/s10803-013-1814-y

"Data collected at the University of Kentucky. Victoria Knight will be at Vanderbilt in Fall 2013".

The online version of the original article can be found under doi:10.1007/s10803-013-1814-y.

V. Knight

Peabody College, Vanderbilt University, Nashville, TN, USA

B. R. McKissick ( $\square)$

Mississippi State University, 310 Allen Hall, Box 9705,

Starkville, MS 39762, USA

e-mail: bmckissick@colled.msstate.edu

A. Saunders

University North Carolina at Charlotte, Charlotte, NC, USA 